\title{
Water Vapor Endometrial Ablation for Heavy Menstrual Bleeding: 36-Month Follow-Up of a Prospective, Multicenter Pivotal Clinical Trial
}

This article was published in the following Dove Press journal: International Journal of Women's Health

\author{
Nicholas Leyland (D)' \\ Micah Harris $\mathbb{D}^{2}$ \\ 'Department of Obstetrics and \\ Gynaecology, Michael G. DeGroote \\ School of Medicine, McMaster University, \\ Hamilton, ON, L8S 4KI, Canada; \\ ${ }^{2}$ AEGEA Medical, Menlo Park, CA, \\ 94025, USA
}

Study Objective: To report 36-month follow-up of a pivotal trial that evaluated the safety and effectiveness of the AEGEA Water Vapor System for the treatment of heavy menstrual bleeding (HMB) secondary to Abnormal Uterine Bleeding due to Endometrial disorders or Leiomyomata (AUB-E and AUB-L).

Methods: A prospective, multicenter, single-arm clinical trial at 14 sites in the US, Canada, Mexico, and the Netherlands. Inclusion criteria included a Pictorial Blood Loss Assessment (PBLAC, Higham) score $\geq 150$ and allowed treatment of subjects with leiomyomata classified by the International Federation of Gynecology and Obstetrics (FIGO) Types 2-6 up to $4 \mathrm{~cm}$ in diameter, a uterine cavity up to $12 \mathrm{~cm}$ in length (uterine sound), Essure ${ }^{\circledR}$ contraceptive inserts and/or prior cesarean section. Follow-up assessments were conducted annually up to 36 months after endometrial ablation. The following outcomes were evaluated for 125/155 women: gynecological adverse events, qualitative assessment of menstrual flow, quality of life using the Menorrhagia Impact Questionnaire (MIQ), patient satisfaction, and medical or surgical reintervention for AUB.

Results: One hundred and fifty-five premenopausal women aged 30 to 50 years were enrolled from September 2014 through May 2015. Water vapor endometrial ablation was performed under varying anesthesia/sedation regimens in offices, surgical centers and operating rooms. There were 6 procedure-related adverse events that occurred between 12- and 36-month follow-up, 1 of which was deemed serious (hematometra managed successfully hysteroscopically). Seventy-two percent reported amenorrhea or light menstrual flow. The mean quality of life (MIQ) score improved from 14.7 at baseline to 6.4. Ninety-three percent reported "Satisfied" or "Very Satisfied." Post-ablation hysterectomy for any indication was performed in $6.5 \%$ of patients, and the total rate of re-intervention for bleeding was $7.1 \%$.

Conclusion: Outcomes 36-months after water vapor endometrial ablation for HMB are consistent with 12- and 24-month follow-up results in all subgroups evaluated. The AEGEA Water Vapor System increases the population of patients amenable to efficacious and acceptable treatment of Heavy Menstrual Bleeding (HMB) due to Abnormal Uterine Bleeding (AUB-E,-L). CinicalTrials.gov NCT01979861 registered November 8, 2013.

Keywords: ablation, endometrial, menorrhagia, vapor

\section{Introduction}

Heavy Menstrual Bleeding (HMB) due to Abnormal Uterine Bleeding (AUB-E,-L) is a significant burden to women world-wide, accounting for one-third of all visits to health-care providers. ${ }^{1}$ Hysterectomy, while a definitive treatment, is associated with increased morbidity, longer recovery and a significantly higher cost to
Correspondence: Micah Harris

Tel + l 602-828-0492

Email micahharris697@msn.com

International Journal of Women's Health 2021:13 169-176 
a health-care system than other, less invasive options. ${ }^{2}$ Non-resectoscopic endometrial ablation as an alternative to hysterectomy is a minimally invasive treatment that may be performed in an office setting, with very low morbidity, shorter procedure time and faster recovery; all of these aspects are favorable to physicians, their patients, and the health-care system as a whole. ${ }^{3}$ Moreover, gynecologists are often closely involved in managing the ongoing care of their patients as primary care providers. ${ }^{4}$ Therefore, long-term safety and effectiveness data (beyond the 12-month follow-up for an endometrial ablation device to earn FDA market-approval) is valuable information for gynecologists as they evaluate treatment options and their longer term implications for their patients.

The AEGEA Water Vapor Ablation System (AEGEA Medical, Menlo Park CA) is an FDA-approved endometrial ablation system that utilizes the rapid energy transfer of water vapor as it changes state to liquid droplets. With a short treatment time of 120 seconds, two automated preprocedure safety tests, and maintenance of a low uterine pressure $(52 \mathrm{~mm} \mathrm{Hg})$, office treatment was possible without the use of complex or general anesthesia regimens. This report provides long term follow-up data post water vapor endometrial ablation with regard to safety, menstrual status, quality of life and patient satisfaction, as well as hysterectomy rates in subjects, including those with nonobstructing FIGO Type 2-6 leiomyomata up to $4 \mathrm{~cm}$ in diameter, a uterine cavity length up to $12 \mathrm{~cm}$, Essure ${ }^{\circledR}$ Permanent Birth Control devices (Bayer, Whippany NJ) and/or prior cesarean section. Twelve-month safety and effectiveness have been previously published for the AEGEA pivotal clinical trial. ${ }^{5}$

\section{Role of the Funding Source}

All research-related financial support was supplied by AEGEA Medical. The authors had access to relevant aggregated study data and other information (such as study protocol, analytic plan and report, validated data table, and clinical study report) required to understand and report research findings. The authors take responsibility for the presentation and publication of the research findings, have been fully involved at all stages of publication and presentation development, and are willing to take public responsibility for all aspects of the work. All individuals included as authors and contributors who made substantial intellectual contributions to the research, data analysis, and publication or presentation development are listed appropriately. The role of the sponsor in the design, execution, analysis, reporting, and funding is fully disclosed. The authors' personal interests, financial or nonfinancial, relating to this research and its publication have been disclosed.

\section{Materials and Methods}

This study was designed as a prospective, multicenter, open label, interventional clinical trial (CinicalTrials.gov NCT01979861). The objective of this study was to evaluate the safety and effectiveness of the Water Vapor Ablation System for the treatment of excessive menstrual bleeding to support a Premarket Approval (PMA) application to the US Food and Drug Administration (FDA). The first subject was treated in September 2014 and 12-month data collection was completed in June 2016. All women signed informed consent and were enrolled at fifteen clinical sites throughout the US, Latin America, the Netherlands and Canada. The study was conducted in accordance with Good Clinical Practice guidelines as contained in the International Conference on Harmonization and US Code of Federal Regulations, and in accordance with the Declaration of Helsinki. The study protocol was approved by both central and local Institutional Review Boards (Copernicus Group, Western IRB, Mercy Hospital IRB, Baylor Scott and White Research Institute IRB, Facultad de Medicina y Hospital UANL, Biomedical Research Alliance of New York IRB, Hamilton Integrated Research Board (Canada), Medical Ethics Committee Isala, the Netherlands). Written informed consent was obtained from all study participants.

The Water Vapor Ablation System is comprised of a reusable Water Vapor Generator and a single-use Water Vapor Probe. The Water Vapor Probe tip diameter is $5.8 \mathrm{~mm}$, and is inserted transcervically into the uterine cavity. Depth of initial insertion is based upon a uterine length measured with a uterine sound. Three specialized balloons are inflated to seal and protect the cervix and anchor the device tip just beyond the endocervix, obviating the need to touch the fundus for positioning. Two safety checks are automatically and sequentially performed with sterile saline to ensure that there are no leaks from the uterus through which water vapor can escape (the Integrity Test) and that the Water Vapor Probe is patent and positioned correctly (the Patency Test). Only then can water vapor treatment be initiated. The 120 -second treatment is preceded by a 20 -second "flush," as water vapor displaces the saline used in the safety tests. Water vapor is continuously generated and 
circulated within the uterine cavity and eventually exits through an outflow lumen in the Water Vapor Probe. The Water Vapor Generator provides continuous real-time monitoring of balloon pressures and cervical temperature and regulates to an intra-uterine pressure of $52 \mathrm{~mm} \mathrm{Hg}$, well below the documented opening pressure of the Fallopian Tubes at $70 \mathrm{~mm} \mathrm{Hg} .{ }^{6}$ The balloons automatically deflate at the end of the treatment.

Subjects were enrolled in the pivotal clinical trial based upon a Pictorial Blood Loss Assessment Chart (PBLAC, Higham) $\geq 150 .^{7}$ The criterion for treatment success was a PBLAC score of $\leq 75$ at 12 months. Subjects were followed to 36 months, with phone interviews conducted at 24- and 36-months post-treatment. Safety endpoints included gynecologic adverse events. Effectiveness endpoints included the administration of the Menstrual Impact Questionnaire (MIQ), the total score of which includes an assessment of menstrual status (amenorrhea, light, moderate, heavy or very heavy bleeding), quality of life, and subject satisfaction. ${ }^{8}$ Also recorded were medical or surgical interventions to treat $\mathrm{AUB}$, and the rate of hysterectomy.

The data were entered into a secure electronic data capture system database (Medrio, San Francisco, CA) by the investigators at each site or by their designee. Categorical data for the study were summarized using frequency tables, with a presentation of the subject counts and percent of subjects. For continuous variables, descriptive statistics include the number of subjects (n), mean, standard deviation, median, minimum, and maximum.

\section{Results}

One hundred fifty-five (155) Intent-to-Treat (ITT) subjects were enrolled in the pivotal clinical study. One hundred and forty-one subjects were available for continued evaluation after 12 months. Eighty-one percent (125/155) completed follow-up to 36 months, with only 3 subjects $(1.9 \%)$ lost to follow-up over the duration of the trial. Subject disposition is provided in Table 1.

The mean age of subjects at baseline was $39.8 \pm 5.2$ (median 40) years. The mean BMI was $30.0 \pm 7.4$ (median 29) $\mathrm{kg} / \mathrm{m}^{2}$. There were 19 (13.4\% 19/141) subjects who reported any gynecologic adverse events $>12-24$ months following ablation and $24(18 \%, 24 / 133)$ subjects who reported any gynecologic adverse events $>24-36$ months following ablation.

During >12-24-month follow-up, there were 2 procedure-related adverse events (AUB and cyclic cramping)
Table I Study Subject Disposition

\begin{tabular}{|c|c|}
\hline & $\mathbf{N}$ \\
\hline Pivotal Clinical Study ITT Analysis Cohort: Vapor & 155 \\
\hline \multicolumn{2}{|l|}{ Ablation Attempted } \\
\hline No treatment received & \\
\hline Subject did not pass Integrity Test & -2 \\
\hline Subject did not pass Patency Test & -4 \\
\hline Incomplete Treatment & -2 \\
\hline Lost to follow-up & -1 \\
\hline Suicide & -1 \\
\hline Hysterectomy for pain (Ovarian Cyst) & -1 \\
\hline IUD for $A U B$ & -1 \\
\hline Major protocol deviations & -2 \\
\hline I 2-Month Follow-up Cohort & 141 \\
\hline Hysterectomy for $\mathrm{AUB}^{\mathrm{a}}$ & -4 \\
\hline Repeat ablation for $\mathrm{AUB}^{\mathrm{a}}$ & -1 \\
\hline Laparoscopy, operative hysteroscopy and hysteroscopic & -1 \\
\hline ablation for endometriosis ${ }^{\mathrm{a}}$ & \\
\hline Lost to follow-up & -1 \\
\hline Voluntary withdrawal & -1 \\
\hline 24-Month Follow-up Cohort & 133 \\
\hline Hysterectomy for AUB & -2 \\
\hline Hysterectomy for pelvic pain & -3 \\
\hline Mirena IUD placement for AUB & -2 \\
\hline Lost to follow-up & -2 \\
\hline $\begin{array}{l}\text { Subject lost-to-follow-up at } 12 \text { and } 24 \text { months, returned } \\
\text { prior to } 36 \text {-month follow-up }\end{array}$ & +1 \\
\hline 36-Month Follow-up Cohort & 125 \\
\hline
\end{tabular}

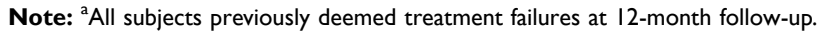

and 5 Serious Adverse Events (SAEs), one of which was procedure-related (hematometra, managed with hysteroscopic lysis of adhesions; the subject continued to participate in the trial to 36 months). The remaining 4 SAE's consisted of: hospitalization for a ruptured ovarian cyst, no intervention taken; and three hysterectomies due to AUB in subjects who did not respond to treatment at 12 months. During >24-36-month follow-up, there were 3 procedurerelated adverse events (cramping and AUB), and 3 SAEs, none of which were deemed related to the procedure (three hysterectomies in subjects not responding to treatment at 12 months). Reintervention for bleeding and hysterectomies are reported separately below. The percent of subjects with severe adverse events for the 12-24 month and 24 to 36 -month periods was $0.7 \%(1 / 141,95 \%$ CI $0.04-$ $3.9 \%)$ and $2.3 \%(3 / 133,95 \%$ CI $0.47-6.5 \%)$ respectively.

Menstrual status at 12-, 24- and 36-month follow-up was reported as none (amenorrhea), light, moderate, heavy or 
Table 2 Menstrual Status at 12, 24, and 36 Months Post-Ablation

\begin{tabular}{|l|l|l|l|}
\hline $\begin{array}{l}\text { Menstrual } \\
\text { Status }\end{array}$ & $\begin{array}{l}\text { Month I2, } \\
\text { N=I4 I }\end{array}$ & $\begin{array}{l}\text { Month 24, } \\
\text { N=I33 }\end{array}$ & $\begin{array}{l}\text { Month 36, } \\
\text { N=I 25 }\end{array}$ \\
\hline Amenorrhea & $20.6 \%(29)$ & $24.8 \%(33)$ & $23.2 \%(29)$ \\
Light & $46.8 \%(66)$ & $50.4 \%(67)$ & $48.8 \%(6 I)$ \\
Moderate & $25.5 \%(36)$ & $19.5 \%(26)$ & $22.4 \%(28)$ \\
Heavy & $6.4 \%(9)$ & $3.8 \%(5)$ & $3.2 \%(4)$ \\
Very Heavy & $0.7 \%(1)$ & $1.5 \%(2)$ & $2.4 \%(3)$ \\
\hline
\end{tabular}

very heavy based on the MIQ. Data presented in Table 2 represents the results based on the total number of subjects who responded to the questionnaire. Figure 1 depicts the sustained effect on bleeding over 36 months. Although Follicle Stimulating Hormone levels were not measured, at 24 months $2 / 141$ subjects $(1.4 \%, 95 \%$ CI $0.17-5.0 \%)$ noted complaints potentially attributable to menopause (vaginal dryness and night sweats). At 36 months an additional 2/ 133 subjects $(1.5 \%, 95 \%$ CI $0.18-5.3 \%)$ complained of "menopausal symptoms." None of these 4 subjects concurrently reported amenorrhea despite these observations.

The Menorrhagia Impact Questionnaire (MIQ) was administered at baseline and at 12-, 24-, and 36-month follow-up to assess quality of life. The worst possible score is 21 , and the best possible score is 4 for this questionnaire. The baseline mean score of 14.7 improved to a mean score of 6.6, 6.1 and 6.3 at 12-, 24-, and 36-month follow-up, respectively. These data are presented below in Table 3 .

Subjects were asked to rate their overall satisfaction with the water vapor ablation procedure. At 36 months, 91.2\% (114/125, 95\% CI 84.8-95.5\%) were satisfied or very satisfied, and $94.4 \%(118 / 125,95 \%$ CI $88.8-97.7 \%)$ would recommend the procedure to a friend.

Eleven subjects (11/155 or $7.1 \%, 95 \%$ CI $3.6-12.3 \%)$ required medical or surgical intervention to treat AUB: 2 had repeat ablations, 3 had progestin-IUDs inserted, and 6 underwent hysterectomy. Over the 36-month duration of the trial, there were $10(10 / 155,6.5 \%, 95 \%$ CI $3.1-11.5 \%)$ reported hysterectomies. The reasons for hysterectomy were AUB $(\mathrm{N}=6)$ and pain $(\mathrm{N}=4)$. One subject was diagnosed with hematometra, and was treated hysteroscopically. Sixty percent $(6 / 10)$ of the hysterectomies had pathology findings of adenomyosis. Additionally, 40\% (4/10) did not meet the study success criteria of PBLAC $\leq 75$ at the 12-month follow-up visit and thus the progression to hysterectomy did not necessarily indicate a worsening of an initial good response to treatment.

This cohort of subjects in the pivotal trial included women with FIGO Types 2-6 Leiomyomata up to $4 \mathrm{~cm}$ in diameter $(\mathrm{n}=21)$, a uterine cavity length $10-12 \mathrm{~cm}(\mathrm{n}=27)$, Essure contraceptive inserts $(\mathrm{n}=5)$, both leiomyomata and

60

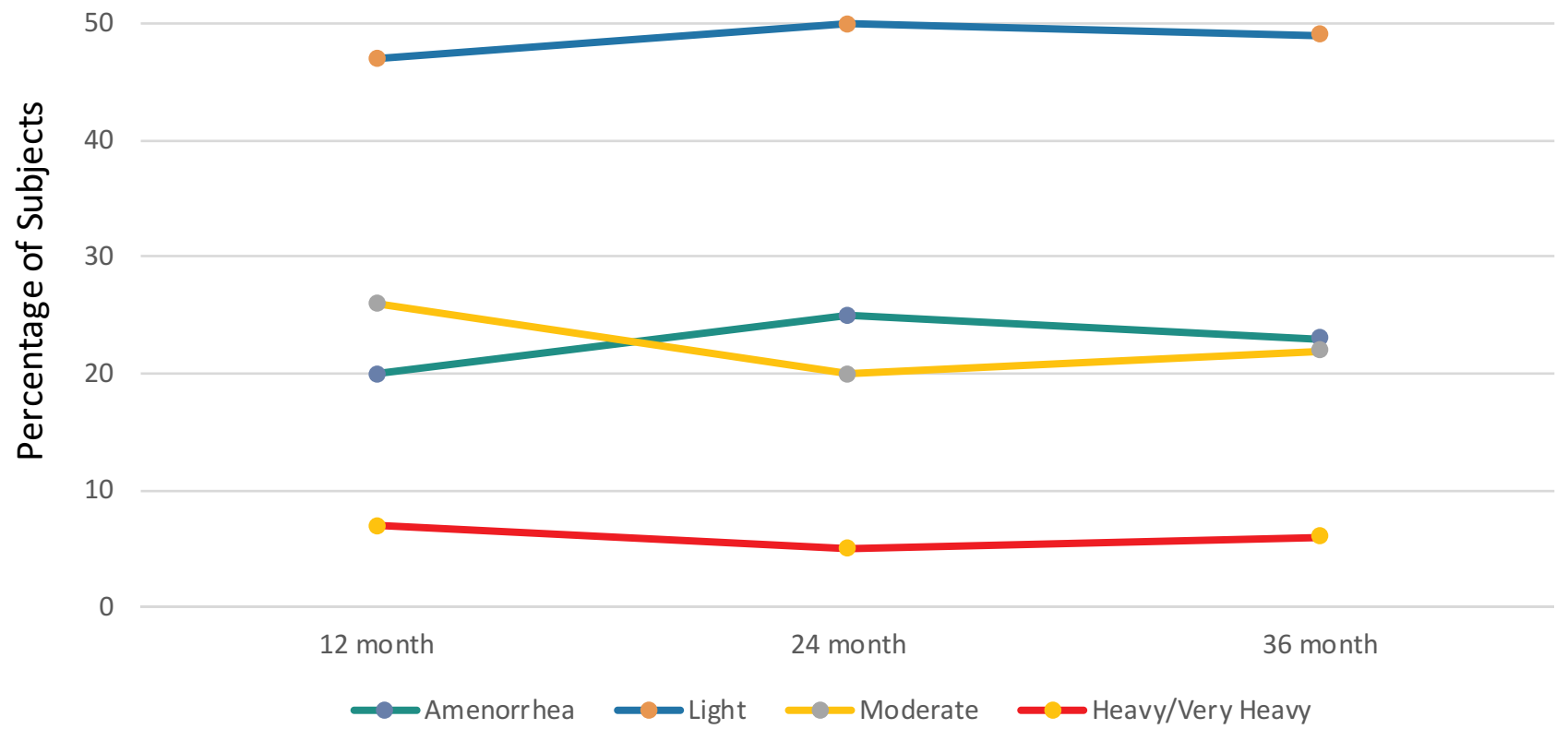

Figure I Menstrual status at 12, 24, and 36 months post-ablation. 
Table 3 Quality of Life Improvement (MIQ)

\begin{tabular}{|l|l|l|l|l|}
\hline MIQ Score & Baseline (N=I4I) & Month I2 (N=I4I) & Month 24 (N=I33) & Month 36 (N=I25) \\
\hline Mean \pm SD (median) & $14.7 \pm 2.9(15.0)$ & $6.6 \pm .1 .8(6.0)$ & $6.1 \pm .1 .7(6.0)$ & $6.3 \pm .1 .9(6.0)$ \\
Range (min, max) & $(6,2 I)$ & $(4,15)$ & $(4,16)$ & $(4,17)$ \\
$95 \% \mathrm{Cl}$ Cl of mean change from baseline & & $-8.7,-7.6$ & $-9.1,-8.0$ & $-9.0,-7.8$ \\
\hline
\end{tabular}

a longer length cavity $(\mathrm{n}=3)$, and both leiomyomata and Essure $(n=1)$. These sub-groups are traditionally outside of the labeling of FDA-approved endometrial ablation devices, and the combined groups represented $45 \%(56 / 125)$ of the patients at 36-month follow-up. The menstrual status outcomes for these subgroups of patients do not differ in comparison to the entire cohort of subjects, with the majority reporting amenorrhea or light bleeding at 36-month followup (Table 4). Regarding reintervention and hysterectomy rates, one subject with both leiomyomata and a uterine length of $10 \mathrm{~cm}$ underwent IUD insertion to treat recurrent AUB; 1 subject with a uterine length of $10 \mathrm{~cm}$ had hematometra; and 1 subject with uterine cavity length of $11 \mathrm{~cm}$ underwent hysterectomy for recurrent AUB. Only 2/10 of the subjects who underwent hysterectomy were from these subgroups, with uterine cavity lengths of 10 and $11 \mathrm{~cm}$.

As previously reported, subjects with a prior cesarean section had similar 12-month success rates to those with normal vaginal deliveries, and had no difference in reported adverse events. ${ }^{5}$ These subjects comprised $41 \%(51 / 125)$ of those available for evaluation at 36 months. Bleeding status in this 36-month cohort of subjects having had 1 or more cesarean sections was reported as amenorrhea in 33\% (17/ $51)$, light in $47 \%(24 / 51)$, moderate in $18 \%(9 / 51)$ and heavy in $2 \%(1 / 51)$, comparable to the entire population. Of the subjects who required reintervention for AUB, those with prior cesarean section comprised $45 \%$ compared to $55 \%$ without cesarean (RR 1.09, 95\% CI 0.3488-3.4343, $\mathrm{P}=0.88$ ). Of the group of subjects who underwent hysterectomy for any indication, subjects with prior cesarean section comprised $60 \%$ compared to $40 \%$ in the non-cesarean cohort. (RR 1.97, 95\% CI 0.579-6.704, $\mathrm{P}=0.28$ ).

\section{Discussion}

This prospective clinical trial demonstrates that the safety and effectiveness of water vapor endometrial ablation is sustained through 36-month follow-up. Bleeding status remained stable as did patient satisfaction throughout the duration of the study. Medical and surgical re-intervention to treat recurrent AUB was low, as was the overall rate of hysterectomy. These results were consistent in subjects with uterine leiomyomata (FIGO Type 2-6 up to $4 \mathrm{~cm}$ ), a longer uterine cavity (up to $12 \mathrm{~cm}$ ) and/or intratubal contraceptive inserts, each of which has been traditionally excluded from endometrial ablation treatment.

Given the importance of long-term data for endometrial ablation procedures, other studies have evaluated the overall risk of hysterectomy or the need for reintervention due to recurrent AUB after endometrial ablation. In a large retrospective analysis of 5591 women in Finland who underwent endometrial ablation with any technique during the period 1997-2014, 19.8\% (1086/5591) of women underwent hysterectomy during the follow-up period, compared to $9.4 \%(2521 / 26,938)$ of controls who had not had endometrial ablation. The adjusted hazard ratio (HR) for hysterectomy for any indication compared to controls who had not had ablation was 3.63 (95\% CI 3.32-3.96, $\mathrm{P}<0.001)$. The risk was highest in the first year following the endometrial ablation and decreased during follow-up. ${ }^{9}$ Three large retrospective cohort studies report

Table 4 Menstrual Status in Subgroups at 36 Months

\begin{tabular}{|c|c|c|c|c|}
\hline $\begin{array}{l}\text { Menstrual Status at 36-Month } \\
\text { Follow-Up }\end{array}$ & $\begin{array}{l}\text { Leiomyoma, } \\
\mathrm{N}=\mathbf{2} \text { I }\end{array}$ & $\begin{array}{l}\text { Cavity Length } \\
10-12 \mathrm{~cm}, \mathrm{~N}=27\end{array}$ & $\begin{array}{l}\text { Leiomyoma and Cavity Length } \\
10-12 \mathrm{~cm}, \mathrm{~N}=3\end{array}$ & $\begin{array}{l}\text { Essure Only, } \\
N=5\end{array}$ \\
\hline Amenorrhea & $28.6 \%(6 / 21)$ & $25.9 \%(7 / 27)$ & 0 & $20 \%(1 / 5)$ \\
\hline Light & $47.6 \%(10 / 21)$ & $48.1 \%(13 / 27)$ & $67 \%(2 / 3)$ & $80 \%(4 / 5)$ \\
\hline Moderate & $23.8 \%(5 / 21)$ & $11.1 \%(3 / 27)$ & $33 \%(1 / 3)$ & 0 \\
\hline Heavy & 0 & $7.4 \%(2 / 27)$ & 0 & 0 \\
\hline Very Heavy & 0 & $7.4 \%(2 / 27)$ & 0 & 0 \\
\hline
\end{tabular}


hysterectomy rates for the indication of failed endometrial ablation ranging between $13 \%$ and $21 \%$, with the majority of hysterectomies performed within 3 years of the endometrial ablation. ${ }^{10-12}$ In these studies, resectoscopic, thermal balloon or radiofrequency devices were used. Prospective studies comparing endometrial ablation with thermal balloon or hysteroscopic resection to hysterectomy suggest a higher incidence of re-intervention within the ablation group within the first 3 years of follow-up, reporting rates $31-38 \%$ by 4 years. ${ }^{13,14}$ The present study reports 36-month hysterectomy and re-intervention rates that are much lower at $6.5 \%$ and $7.1 \%$, respectively.

The effects of leiomyomata, submucous or otherwise, on the long-term effectiveness of endometrial ablation in the treatment of AUB have been studied. Lybol et al reported retrospective data on 486 subjects who had undergone radiofrequency endometrial ablation from 2008 to $2014 .^{15} 18.3 \%$ of subjects required medical or surgical reintervention for recurrent HMB or cyclic pain; $62.2 \%$ presented with complaints within 1 year and $83.3 \%$ within 2 years. In addition to younger age, a history of sterilization, and a history of dysmenorrhea, which have been shown in multiple studies to be associated with endometrial ablation failure, an intramural leiomyoma noted by ultrasound carried an increased Odds Ratio (OR) of 2.23 (95\% CI 1.27-3.93, $\mathrm{P}=0.005$ ). Twenty-four subjects with FIGO Types 2-6 leiomyomata were treated in the pivotal study and did not note a deleterious effect on bleeding, reintervention or satisfaction compared to the entire cohort. It has been traditionally acknowledged that the treatment of $\mathrm{HMB}$ due to AUB-L in the presence of submucous leiomyomata is better undertaken with resection rather than endometrial ablation alone.

FDA-approved labeling for current endometrial ablation devices does not include treatment of cavities ranging in uterine sound length greater than a maximum of 10 to $10.5 \mathrm{~cm} .{ }^{16-20}$ Peeters et al evaluated 76 of 890 women who underwent either radiofrequency ablation, ablation with circulating hot water, or a thermal balloon and subsequently required reintervention; they were compared to 76 controls with a successful procedure. ${ }^{21}$ Factors associated with a failure of the ablation procedure included a history of dysmenorrhea, a submucous myoma and longer uterine length. Regarding uterine length, the risk of ablation failure increased with each additional centimeter in length above a mean of $8.77 \mathrm{~cm}$ (OR $1.3695 \% \mathrm{CI}$ $1.05-1.75, \mathrm{P}<0.018$ ). Also, in this study, as with the study reported here, subjects with prior cesarean section showed no difference in safety or effectiveness compared to controls (OR 0.76, 95\% CI 0.3-1.8).

In a comprehensive review of the literature surrounding endometrial ablation and Essure Permanent Birth Control, there is no evidence that the tubal inserts increase the potential for injury or impact effectiveness of either endometrial ablation or sterilization with most currently or previously available devices. ${ }^{22}$ In this trial, each of the 8 subjects with Essure devices was required to have the 3-month confirmation test to demonstrate tubal occlusion prior to inclusion in the pivotal trial. No Essure devices were placed concurrently with endometrial ablation, and no significant device-related adverse events were noted. Six of the 8 subjects in the ITT cohort progressed to 36month follow-up. No decrease in effectiveness was seen; one subject was lost to follow-up at 24 months, having reported treatment success and satisfaction at 12 months, and one did not pass the pre-procedure safety test and did not undergo treatment.

One limitation of this study is that the number of subjects in the subgroups with leiomyomata, a uterine cavity length 10-12 cm, and Essure contraceptive inserts is small, and larger populations would be required to make definitive statements about results in these groups. Strengths of the study include its prospective design and high rate of subject retention through 3 years of follow-up.

\section{Conclusion}

Long-term follow-up results from this prospective, international multi-center clinical trial indicate that safety, menstrual status, quality of life and patient satisfaction are stable 36 months following water vapor endometrial ablation. Even with the inclusion in the clinical trial of subjects with traditionally challenging anatomic characteristics, the need for re-intervention or hysterectomy is low at 36-month follow-up when compared to many different endometrial ablation reports in the literature.

\section{Data Sharing Statement}

Will individual participant data be available (including data dictionaries)? NO. What data in particular will be shared? N/ A. What other documents will be available? N/A. When will data be available (start and end dates)? N/A. By what access criteria will data be shared (including with whom, for what types of analyses, and by what mechanism)? N/A. 


\section{Acknowledgments}

The AEGEA Investigator Group Jeffrey Baker M.D., Clinical Research Prime, Idaho Falls, ID. Cindy Basinski M.D., CMB Research, Newburgh, IN. Jose G Garza-Leal M.D., Hospital Universitario Dr. Jose E Gonzalez Universidad Autónoma de Nuevo León, Monterrey, MX. Seth Herbst M.D., Institute for Women's Health and Body, Wellington, FL. D. Alan Johns M.D., Baylor, Scott and White Research Institute Fort Worth, TX. Mark Levie, M.D., Montefiore Medical Center, Bronx, NY. David Levine M.D., Mercy Hospital, St. Louis, MO. Andrea Lukes M.D. Carolina Women's Research and Wellness, Durham, NC. Charles Miller M.D., The Advanced Gynecologic Surgery Inst., Naperville, IL. James Presthus M.D., Fairview Center for Women, Edina, MN. Christopher Walker M.D., Clinical Associates of Orlando, Orlando, FL. Hugo van Eijndhoven, M.D. Isala Hospital, Zwolle, The Netherlands. Kirk Brody M.D., Chattanooga Medical Research LLC, Chattanooga, TN. Statistician Peter Shabe Advance Research Associates, 2350 Mission College Boulevard, Suite 825, Santa Clara, CA 95054. Portions of this manuscript were presented at the 47th AAGL Global Conference In Las Vegas, NV in November 11-15, 2018.

\section{Funding}

This study was sponsored by AEGEA Medical, Menlo Park, CA.

\section{Disclosures}

The authors received research-related support for the conduct of this study. Dr. Harris is Medical Director of AEGEA Medical. Dr Harris reports being a clinical trial investigator for Sebela Pharmaceuticals, Medicines360, Bayer, Inovio Pharmaceuticals, and Myovant, outside the submitted work. Dr Nicholas Leyland reports a research grant from and advisory board and speaker's bureau for AbbVie, outside the submitted work. The authors report no other potential conflicts of interest for this work.

\section{References}

1. Diagnosis of abnormal uterine bleeding in reproductive-aged women. ACOG Practice Bulletin No.128. July, 2012.

2. Miller JD, Lenhart GM, Bonafede M, Basinski CM, Lukes AS, Troeger KA. Cost effectiveness of endometrial ablation with the NovaSure system vs other global ablation modalities and hysterectomy for treatment of abnormal bleeding: US commercial and medicaid payer perspectives. Int J Womens Health. 2015;7:59-73. doi:10.2147/ IJWH.S75030
3. Fergusson RJ, Lethaby A, Shepperde S, Farquhar C. Endometrial resection and ablation versus hysterectomy for heavy menstrual bleeding (review). Cochrane Database Syst Rev. 2013;11: CD000329.pub2.

4. Mazzoni S, Brewer S, Durfee J, et al. Patient perspectives of obstetrician-gynecologists as primary care providers. J Reprod Med. 2017;62(1-2):3-8.

5. Levie MD, Chudnoff SG. A prospective multicenter pivotal trial to evaluate the safety and effectiveness of the AEGEA vapor endometrial ablation system. J Minim Invasive Gynecol. 2019;26 (4):679-687. doi:10.1016/j.jmig.2018.07.012

6. Baker VL, Adamson GD. Threshold intrauterine perfusion pressures for intraperitoneal spill during hydrotubation and correlation with tubal adhesive disease. Fertil Steril. 1995;64(6):1066-1069. doi:10.1016/S0015-0282(16)57961-8

7. Higham JM, O'Brien PM, Shaw RW. Assessment of menstrual blood loss using a pictorial chart. Br J Obstet Gynaecol. 1990;97 (8):734-739. doi:10.1111/j.1471-0528.1990.tb16249.x

8. Bushnell DM, Martin ML, Moore KA, Richter HE, Rubin A, Patrick DL. Menorrhagia impact questionnaire: assessing the influence of heavy menstrual bleeding on quality of life. Curr Med Res Opin. 2010;26(12):2745-2755. doi:10.1185/03007995.2010.532200

9. Soini T, Rantanen M, Paavonen J, et al. Long-term follow-up after endometrial ablation in Finland. Obstet Gynecol. 2017;130:554-560. doi:10.1097/AOG.0000000000002166

10. Longinotti MK, Jacobson GF, Hung YY, Learman LA. Probability of hysterectomy after endometrial ablation. Obstet Gynecol. 2008;112 (6):1214-1220. doi:10.1097/AOG.0b013e31818c1766

11. Shavell VI, Diamond MP, Senter JP, Kruger ML, Johns DA. Hysterectomy subsequent to endometrial ablation. J Minim Invasive Gynecol. 2012;19(4):459-464. doi:10.1016/j.jmig.2012.03.013

12. El Nashar E, Hopkins M, Creedon D, et al. Prediction of treatment outcomes after global endometrial ablation. Obstet Gynecol. 2009;113(1):97-106. doi:10.1097/AOG.0b013e31818f5a8d

13. Dickersin K, Munro M, Clark M, et al. Hysterectomy compared with endometrial ablation for dysfunctional uterine bleeding. Obstet Gynecol. 2007;110(6):1279-1289. doi:10.1097/01.AOG.0000292 083.97478 .38

14. Aberdeen Endometrial Ablation Trials Group. A randomised trial of endometrial ablation versus hysterectomy for the treatment of dysfunctional uterine bleeding: outcome at 4 years. $\mathrm{Br} J$ Obstet Gynaecol. 1999;106:360-366. doi:10.1111/j.1471-0528.1999. tb08275.x

15. Lybol C, van der Coelen S, Hamelink A, et al. Predictors of long-term novasure endometrial ablation failure. J Minim Invasive Gynecol. 2018;25(7):1255-1259. doi:10.1016/j.jmig.2018.03.006

16. Novasure ${ }^{\circledR}$ Instructions for Use and Controller Operator's Manual. Marlborough, MA:Hologic Inc.

17. Genesys HTA ${ }^{T M}$ System Instructions for Use. Marlborough MA: Boston Scientific.

18. Minerva Endometrial Ablation System Instructions for Use. Redwood City, CA:Minerva Surgical.

19. Her Option Office Cryoablation Therapy Instructions for Use. Trumbull CT:Cooper Surgical.

20. Cerene ${ }^{\circledR}$ Cryotherapy Device Instructions for Use. Emeryville, CA: Channel Medsystems.

21. Peeters J, Penninx J, Mol B, Bongers M. Prognostic factors for the success of endometrial ablation in the treatment of menorrhagia with special reference to cesarean section. Eur J Obstet Gynecol Reprod Biol. 2013;167:100-103. doi:10.1016/j.ejogrb.2012.11.009

22. Aldape D, Chudnoff S, Levie M. Global endometrial ablation in the presence of essure ${ }^{\circledR}$ microinserts. Rev Obstet Gynecol. 2013;6 (2):80-88 


\section{Publish your work in this journal}

The International Journal of Women's Health is an international, peerreviewed open-access journal publishing original research, reports, editorials, reviews and commentaries on all aspects of women's healthcare including gynecology, obstetrics, and breast cancer. The

manuscript management system is completely online and includes a very quick and fair peer-review system, which is all easy to use. Visit http://www.dovepress.com/testimonials.php to read real quotes from published authors.

Submit your manuscript here: https://www.dovepress.com/international-journal-of-womens-health-journal 